\title{
Revealing Sub-Surface Vibrational Modes by Atom-Resolved Damping Force Spectroscopy
}

\author{
Makoto Ashino, ${ }^{1}$ Roland Wiesendanger, ${ }^{1}$ Andrei N. Khlobystov, ${ }^{2}$ Savas Berber,,${ }^{3,4}$ and David Tománek ${ }^{4}$ \\ ${ }^{1}$ Institute of Applied Physics and Microstructure Research Centre, \\ University of Hamburg, 20355 Hamburg, Germany \\ ${ }^{2}$ School of Chemistry, University of Nottingham, University Park, Nottingham NG7 2RD, UK \\ ${ }^{3}$ Physics Department, Gebze Institute of Technology, 41400 Gebze, Kocaeli, Turkey \\ ${ }_{4}^{4}$ Physics and Astronomy Department, Michigan State University, East Lansing, Michigan 48824-2320, USA
}

(Dated: November 15, 2018)

\begin{abstract}
We propose to use the damping signal of an oscillating cantilever in dynamic atomic force microscopy as a noninvasive tool to study the vibrational structure of the substrate. We present atomically resolved maps of damping in carbon nanotube peapods, capable of identifying the location and packing of enclosed Dy@ $\mathrm{C}_{82}$ molecules as well as local excitations of vibrational modes inside nanotubes of different diameter. We elucidate the physical origin of damping in a microscopic model and provide quantitative interpretation of the observations by calculating the vibrational spectrum and damping of Dy@ $\mathrm{C}_{82}$ inside nanotubes with different diameters using ab initio total energy and molecular dynamics calculations.
\end{abstract}

PACS numbers: 81.05.Tp, 61.48.De, 68.37.Ps 68.35.Ja

Detecting surface and subsurface vibrations with atomic spatial resolution is a daunting endeavor. Reported observations of molecular vibrations by Inelastic Scanning Tunnelling Spectroscopy (IESTS) require an electrically conducting substrate [1]. Atomic Force Microscopy (AFM) experiments involving ultrasonic oscillations of elastically indented samples [2, 3] can be performed on electrically insulating systems, but yield subsurface images with nanoscale resolution at best. Building on the high spatial resolution and sensitivity of dynamic non-contact AFM [4, 5], we introduce Damping Force Spectroscopy (DFS) as a non-invasive tool to study subsurface structure and vibrational modes in complex molecular systems at the atomic scale. We have chosen carbon nanotube peapods [6, 7, 8] consisting of linear chains of fullerenes enclosed in single-wall carbon nanotubes (SWNTs) [9] as a prominent example of supramolecular compounds. Of particular interest are $\left(\mathrm{M} @ \mathrm{C}_{n}\right) @ \mathrm{SWNT}$ peapods containing $\mathrm{M} @ \mathrm{C}_{n}$ metallofullerenes, hollow cages of $n$ carbon atoms surrounding the metal atom $\mathrm{M}$, known for their unusual electronic transport behavior 10.

Here, we demonstrate that monitoring the damping of an oscillating AFM tip provides invaluable information not only about topography, but also the subsurface vibrational modes that have not been observed before with atomic-scale spatial resolution. Our DFS studies of $\left(\mathrm{Dy} @ \mathrm{C}_{82}\right) @ S W N T$ indicate that the observed damping of the tip oscillation depends sensitively on its position and host tube diameter of $\left(\mathrm{Dy}_{\mathrm{C}} \mathrm{C}_{82}\right) @ \mathrm{SWNT}$, in agreement with extensive molecular dynamics (MD) studies reported here. Results of our predictive calculations trace back the observed damping to the excitation of local vibrational modes by transferring energy from the oscillating AFM tip. This truly mechanical oscillator couples to the enclosing nanotube first and subsequently to the enclosed molecules, revealing their packing structure.

$\left(\mathrm{Dy} @ \mathrm{C}_{82}\right) @ S W N T$ peapods were prepared by encapsulating Dy@ $\mathrm{C}_{82}$ metallofullerenes in open-ended SWNTs at a filling rate of $\approx 60 \%$, as confirmed by high-resolution transmission electron microscopy and DFS [11]. The peapods were deposited at low coverage onto an insulating flat $\mathrm{SiO}_{2}$ surface of a $\mathrm{Si}$ substrate and observed by a home-built dynamic AFM [11], shown schematically in Fig. 1(a). The AFM, equipped with a commercial Si cantilever (spring constant of $34.3 \mathrm{~N} / \mathrm{m}$, eigenfrequency of $159 \mathrm{kHz}$ ) and Si tip (nominal tip radius of $20 \AA$ ), was operated at constant oscillation amplitude of $21-23 \AA$ under ultra-high vacuum $\left(p<1 \times 10^{-8} \mathrm{~Pa}\right)$ at low temperature $(T<13 \mathrm{~K})$.

High-resolution AFM topography observations of SWNTs and peapods, illustrated in Fig. 1(a), are shown by contour plots in Fig. 22(a:1-4) [11]. Our topography results indicate that the diameter of the empty SWNT in Fig. 2(a:1) is $d=16.2 \pm 0.5 \AA$. Short-range interatomic interactions, probed by the dynamic AFM, provide atomicscale contrast in topography images [12]. The observed atomic arrangement in this tube identifies its chiral index as $(18,5)$ [13]. Comparing these results to those for a peapod of the same diameter, presented in Fig.2(a:2), we find the atomic-scale topography features to be amplified with respect to the empty SWNT. This contrast enhancement can likely be attributed to local stiffening of the nanotube wall next to the enclosed Dy@ $\mathrm{C}_{82}$ molecules.

The optimum packing geometry of fullerenes in peapods is determined primarily by their diameter $\left(d_{F}\right)$ and that of the enclosing nanotube $(d)[8]$. We expect an optimum snug fit at $d \approx d_{F}+6.4 \AA[14,15]$, with the center-to-center inter-fullerene distance $d_{F-F} \approx d_{F}+$ $3.2 \AA$, nearly independent of the nanotube diameter. 

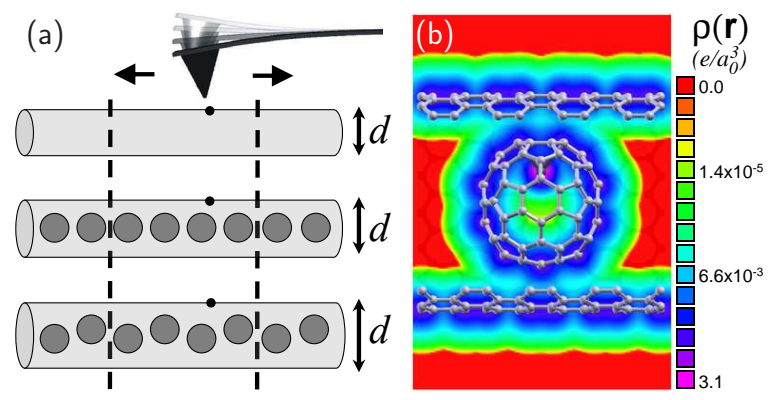

(c)

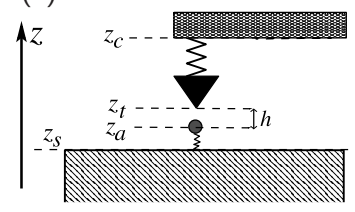

(d) 2

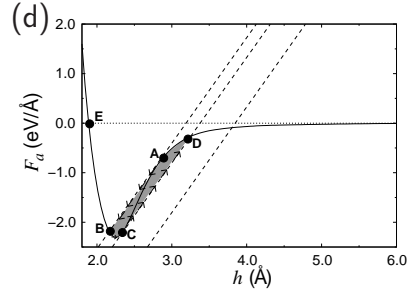

FIG. 1: (Color) (a) Schematic of an oscillating AFM tip scanning across nanotube peapods with different diameters $d$ at different filling levels. (b) Total charge density of the optimized Dy@ $\mathrm{C}_{82}$ metallofullerene encapsulated in the $(18,0)$ nanotube, superposed with the skeletal structure of the peapod. (c) Schematic of the coupling mechanism between a sharp AFM tip and a flexible substrate. The cantilever stiffness is represented by the spring connecting the tip and its suspension. The elastic substrate is represented by the spring connecting a representative atom to its surroundings. (d) Mechanism of energy dissipation during the dynamic AFM operation in non-contact regime.

With the diameter $d_{F} \approx 8.0 \AA$ of $\mathrm{Dy} @ \mathrm{C}_{82}$, a $d \approx 14.2 \AA$ wide SWNT should provide an optimum fit, with the encapsulated molecules separated by $d_{F-F} \approx 11.2 \AA$. The diameter $d=13.0 \pm 0.5 \AA$ of the narrowest peapod observed here, discussed in Fig. 2(a:4), is smaller than optimum. In this case, the enclosed molecules distort locally the nanotube wall [16], as seen in Fig. 2(a:4). We find the observed undulation period of $11.5 \pm 0.5 \AA$ to agree with the above estimated inter-fullerene distance $d_{F-F}$.

More intriguing than the topography are our results for the spatial variation of the energy loss $\Delta E$ per cycle of the cantilever oscillation, defined as damping and monitored in DFS [11]. The damping data, shown in Fig. 2(a:58), have been recorded simultaneously with the topography data [4, 5]. For better quantitative comparison, we present topography and damping data for the same system as line plots in Fig. 2(b). According to Fig. 2(a:5), almost no damping is observed in an empty SWNT. In comparison to these observations, Fig. 2(a:6) suggests that damping increases significantly in a peapod of the same diameter. Decreasing the diameter of the enclosing nanotube causes an increasingly snug fit of $\mathrm{Dy} @ \mathrm{C}_{82}$ in the peapod. Our data in Figs. 2(a:6-8) indicate that (i) $\Delta E$ increases, (ii) the atomic-scale features within $\Delta E$ are amplified, and (iii) helical undulations in $\Delta E$ grow in magnitude with decreasing diameter of the enclosing (a)
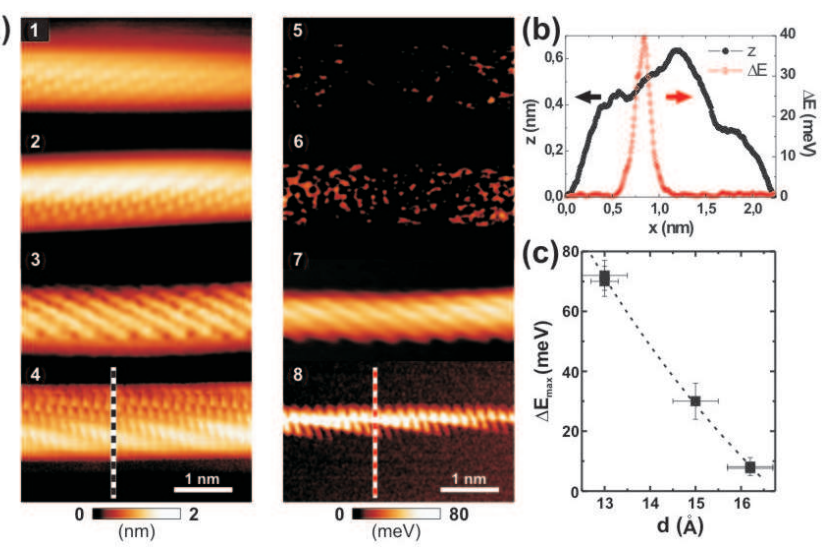

FIG. 2: (Color) AFM topography and damping in SWNTs and peapods of different diameter $d$. (a) Atomically-resolved dynamic AFM observations of the topography (a:1-4) and damping (a:5-8), defined as energy loss per cycle $\Delta E$ and represented by the color-coded 3D contour plots. Results of simultaneous scans for an empty SWNT with $d=16.2 \pm 0.5 \AA$ are shown in (a:1,5), those for peapods with $d=16.2 \pm 0.3 \AA$ in (a:2,6), with $d=15.0 \pm 0.5 \AA$ in (a:3,7), and with $d=$ $13.0 \pm 0.5 \AA$ in $(\mathrm{a}: 4,8)$. (b) Topographic and damping profiles across the same nanotube peapod. The topographic data $z(x)$, represented by the black line, are taken along the blackwhite dotted trace in (a:4). The damping data $\Delta E(x)$, represented by the red circles, are taken along the red-white dotted trace in (a:8). (c) Relationship between nanotube diameter $d$ and the maximum damping energy $\Delta E_{\max }$. The data points are representative for tens of tube samples. The dashed line is a guide to the eye.

nanotube. In the case of a snug fit, atomic-scale features in $\Delta E$ show a similar or even larger contrast than topography features. Even though both are spatially correlated, we observe the strongest damping slightly off the center of the largest topographic protrusions on-top the encapsulated fullerenes.

The topography images in Figs. 2(a) and 2(b) show some lateral asymmetry, which may be due to $\mathrm{Dy} @ \mathrm{C}_{82}$ ordering within the peapod or an artifact [11]. While we have taken precautions to avoid artifacts caused by tip-induced nanotube displacement (we scanned in the axial direction) and feedback lag (we used a small scan velocity), we can not exclude an intrinsically asymmetric shape or doping profile of the AFM tip as the cause of the asymmetry. Irrespective of this finding, we conclude that monitoring the damping of the oscillating tip provides information superior to topography imaging and allows to discriminate between snug and loose packing of fullerenes inside the nanotube.

In Fig. 2(c) we plot the maximum energy loss per cycle $\Delta E_{\max }$ observed during a DFS scan as a function of the host nanotube diameter $d$. The data points in the diagram are presented for a $(13,6)$ and a $(11,8)$ nanotube with $d=13.0 \pm 0.5 \AA$, a $(19,0)$ nanotube with $d=15.0 \pm 0.5 \AA$, and $(20,1)$ and $(18,5)$ nanotubes with 
$d=16.2 \pm 0.5 \AA$. Our results indicate a universal dependence of $\Delta E_{\max }$ on the diameter $d$ only. From the narrowest to the widest nanotube, we find the value of $\Delta E_{\text {max }}$ to decrease from $71 \pm 5 \mathrm{meV}$ to $30 \pm 6 \mathrm{meV}$ and $7.9 \pm 3.0 \mathrm{meV}$ per cycle of the oscillating tip.

To uncover the origin of damping in DFS, we performed $a b$ initio density functional calculations of the electronic structure, equilibrium geometry and elastic response of these systems, along with MD calculations of the dynamical coupling between the AFM tip and the peapod.

Our total energy calculations indicate that unlike the most stable $\mathrm{C}_{2}$ isomer of $\mathrm{C}_{82}$, the near-spherical isomer of $\mathrm{Dy} @ \mathrm{C}_{82}$ has a different $\mathrm{C}_{2 v}$ symmetry. This structure is energetically preferred by at least $0.5 \mathrm{eV}$ over any other isomer and likely abounds in our samples. The total charge distribution of $\mathrm{Dy} @ \mathrm{C}_{82}$ inside the $(18,0)$ nanotube, providing optimum enclosure, is shown in Fig. 1(b).

The fundamental concept of an AFM imaging an elastic substrate is depicted in Fig. 1(c). The position $z_{t}$ of the tip apex atom depends on the position $z_{c}$ of the cantilever mount and the deflection of the elastic cantilever, represented by a spring. Similarly, an elastic substrate is represented by a different spring, allowing the position $z_{a}$ of the closest surface atom to differ from $z_{s}$ of the substrate. The force exerted on the tip by the substrate depends largely on the closest tip-substrate distance $h=z_{t}-z_{a}$.

To elucidate the origin of damping in the dynamical $\mathrm{AFM}$, we first consider the force $F_{a}$ exerted on a substrate carbon atom by an approaching AFM tip at height $h$, shown by the solid line in Fig. 1(d). For a given position $z_{c}$ of the cantilever mount, the instantaneous position of the tip $z_{t}$ and the substrate atom $z_{a}$ depends not only on their mutual distance $h$, which determines the nonlinear interaction force shown by the solid line, but also on the compensating forces of the strained cantilever and substrate, shown by the dotted lines, as well as the history. In the case of a soft cantilever or substrate, as the AFM tip approaches from far away, an instability occurs at point $\mathrm{A}$, causing an abrupt decrease in the tip-substrate distance to point B. During the retraction cycle, a similar instability occurs at C, causing an abrupt increase in the tip-substrate distance to $\mathrm{D}$. We note that even the closest-approach point $\mathrm{B}$ occurs in the noncontact regime, since $h_{B}$ is beyond the equilibrium tipsubstrate separation $h_{E}$, characterized by $F_{a}=0$. The hysteresis corresponding to the shaded area in Fig. $\mathbb{1}(\mathrm{d})$, delimited by A,B,C and D, represents the energy dissipation in the substrate during an ideal approach-retraction cycle.

To quantitatively analyze, how the energy loss of the oscillating tip depends on its position and the tube diameter, we performed a series of MD calculations [11]. We induced an initial perturbation by radially displacing a surface atom by $0.3 \AA$, corresponding to the distance $h_{C}-h_{E}$ in Fig. 1(d), and abruptly releasing it. The observed energy loss per cycle $\Delta E$ depends on the frequency $h \nu$ of vibrational modes excited by the plucking process and the likelihood of exciting them. This energy is then dissipated efficiently owing to the high thermal conductivity of nanotube systems 17]. Since vibrations excited in the cantilever do not change with position, spatial contrast in $\Delta E_{\max }$ is only due to the substrate.

In empty SWNTs, we found that plucking excites only the radial breathing mode (RBM) in the frequency range $h \nu=17.8-23.1 \mathrm{meV}$ in the systems considered here. The energy needed to excite this mode is consistent with $\Delta E$ values seen in Fig. 2(a:5). We expect stronger damping in peapods due to additional modes introduced by the fullerenes. Ultra-soft vibrations of Dy within the $\mathrm{C}_{82}$ cage at $h \nu=4.1-16.5 \mathrm{meV}$ are caused by the soft $\mathrm{Dy}-\mathrm{C}_{82}$ interaction and the large mass of Dy. Since these modes are decoupled from the remaining modes, we will ignore the presence of Dy in the following discussion. Among the fullerene modes, librations about the center are the softest, followed by axial and off-axis motion of the center of mass. Harder fullerene modes include the RBM at $h \nu=29 \mathrm{meV}$, the quadrupolar deformation mode near $h \nu=62.0 \mathrm{meV}$, and higher multipolar modes. Especially in the case of a snug fit in the peapod, the nanotube and fullerene modes are strongly mixed.

For each of the nanotubes studied, we changed the axial position of the surface atom plucked from on-top a fullerene to in-between fullerenes, and also studied a position half-way between the two. Our simulations indicate that damping is strongest for the last position, due to the possibility of exciting librations and axial vibrations of the fullerene underneath. This off-center position, indicated in the inset of Fig. 3(a), agrees with the interpretation of our data in Fig. 2(a).

Our MD simulation results for $\mathrm{C}_{82} @(18,0)$ are presented in Figs. 3(a) and 3(b). The radial trajectory of the plucked atom, $\Delta r(t)$ shown in Fig. B(a), and that of the closest fullerene molecule, $\Delta r_{C M}(t)$ shown in Fig. $3(\mathrm{~b})$, suggest that several modes are excited. To understand which modes dominate damping, we Fourier analyzed all trajectories in our study. The Fourier spectra of $\Delta r(t)$ are shown in Fig. 3(c) and those of $\Delta r_{C M}(t)$ in Fig. 3(d) for zigzag nanotubes ranging from $(17,0)$ to $(21,0)$. Data for intermediate nanotube diameters have been obtained by interpolation.

We consider the narrow $\mathrm{C}_{82} @(17,0)$ peapod to provide optimum coupling between the snugly fit fullerene and the enclosing nanotube. In this case, $\Delta E$ and its spatial modulation should be particularly strong. In contrast to an empty SWNT, the vibration spectrum of a surface atom in $\mathrm{C}_{82} @(17,0)$ is very rich, as seen in Fig. 3(c). Besides low-frequency modes with $h \nu<20.7 \mathrm{meV}$, it contains modes with equally spaced frequencies spanning the range $0<h \nu<90.9 \mathrm{meV}$. In the wider $(18,0)$ nan- 

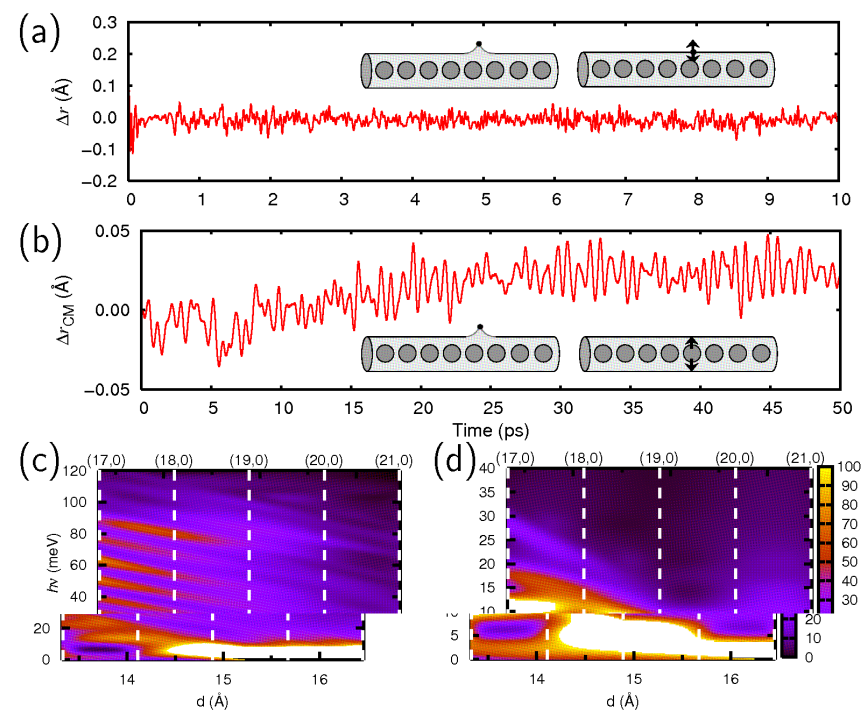

FIG. 3: (Color) Calculated dynamics and energy dissipation in the $\mathrm{C}_{82} @ \mathrm{SWNT}$ system following an initial 'radial plucking' at the nanotube surface. Strongest damping occurs when plucking an atom located axially $\approx 1 \AA$ off the center of the nearest $\mathrm{C}_{82}$. (a) Radial displacement of the plucked atom as a function of time in $\mathrm{C}_{82} @(18,0)$. (b) Radial displacement of the $\mathrm{C}_{82}$ center-of-mass as a function of time in $\mathrm{C}_{82} @(18,0)$. (c) Fourier spectrum of the radial displacement of the initially plucked atom as a function of the nanotube diameter $d$. (d) Fourier spectrum of the $\mathrm{C}_{82}$ center-of-mass as a function of the nanotube diameter $d$. The insets in (a) and (b) illustrate the initial distortion and the quantity monitored.

otube, the vibration spectrum is similar, but softer. In the $(19,0)$ and wider nanotubes, the fullerene-nanotube coupling is reduced drastically, as fullerenes may rearrange at little energy cost. As seen in Fig. 3(c), decreased fullerene-nanotube coupling causes a strong intensity drop of the high-frequency modes at $h \nu>20 \mathrm{meV}$. Especially in the narrow nanotubes, our results for the offcenter plucking position in Fig. 3(c) differ from those of other simulations, where the plucked atom is on top of a fullerene. Since plucking on top a fullerene excites neither librations nor axial fullerene motion, energy dissipation in this plucking position is lower. Comparing the results for the two plucking positions explains the high spatial modulation of $\Delta E$ seen in Fig. 2(a:8).

The vibration spectrum of the center-of-mass motion of a $\mathrm{C}_{82}$ fullerene in the $(17,0)$ nanotube, shown in Fig. 3(d), is rather featureless and much softer than that in Fig. 3(c). The $h \nu \approx 8 \mathrm{meV}$ off-axis vibration mode, which dominates the $(17,0)$ spectrum, red shifts in wider nanotubes. Owing to the zigzag or helical arrangement of fullerenes in wider peapods, the off-axis vibrations of the fullerenes may couple to soft axial vibrations and librations, which appear in the Fourier spectrum of $\Delta r_{C M}$ in Fig. 3(d). These important soft modes, which do not change $\Delta r_{C M}$, modify the spectrum indirectly by coupling anharmonically to off-axis modes. Especially in the wider nanotubes, we find the center-of-mass vibrations of $\mathrm{C}_{82}$ to be rather insensitive to the axial position of the plucked atom, thus contributing very little to the spatial modulation of $\Delta E$.

In conclusion, Damping Force Spectroscopy can reveal geometrical packing and vibrational spectra of subsurface structures at the atomic scale. We expect that this technique will gain significant appeal for atomic-level investigations of three-dimensional supramolecular systems.

We thank Siegmar Roth and Dirk Obergfell for useful discussions and for sample preparation. We gratefully acknowledge financial support from the Deutsche Forschungsgemeinschaft and from the National Science Foundation under NSF-NSEC grant No. 425826 and NSF-NIRT grant No. ECS-0506309. Computational resources have been provided by the Michigan State University High Performance Computing Center.

[1] B. C. Stipe, M. A. Rezaei, and W. Ho, Science 280, 1732 (1998).

[2] K. Yamanaka, H. Ogiso, and O. Kolosov, Appl. Phys. Lett. 64, 178 (1994).

[3] O. V. Kolosov, M. R. Castell, C. D. Marsh, G. A. D. Briggs, T. I. Kamins, and R. S. Williams, Phys. Rev. Lett. 81, 1046 (1998).

[4] S. Morita, R. Wiesendanger, and E. Meyer, Noncontact Atomic Force Microscopy, NanoScience and Technology (Springer, Berlin, 2002).

[5] R. García and R. Pérez, Surf. Sci. Rep. 47, 197 (2002).

[6] B. W. Smith, M. Monthioux, and D. E. Luzzi, Nature 396, 323 (1998).

[7] A. Jorio, G. Dresselhaus, and M. S. Dresselhaus, eds., Carbon Nanotubes: Advanced Topics in the Synthesis, Structure, Properties and Applications, Topics in Applied Physics, Vol. 111 (Springer, Berlin, 2008).

[8] D. A. Britz and A. N. Khlobystov, Chem. Soc. Rev. 35, 637 (2006).

[9] R. Kitaura and H. Shinohara, Chem. Asian J. 1, 646 (2006).

[10] T. Shimada, T. Okazaki, R. Taniguchi, T. Sugai, H. Shinohara, K. Suenaga, Y. Ohno, S. Mizuno, S. Kishimoto, and T. Mizutani, Appl. Phys. Lett. 81, 4067 (2002).

[11] See EPAPS Document No. \#\#\# for experimental and computational details.

[12] M. Ashino, A. Schwarz, T. Behnke, and R. Wiesendanger, Phys. Rev. Lett. 93, 136101 (2004).

[13] M. Ashino and R. Wiesendanger, Jpn. J. Appl. Phys. 45, 2286 (2006).

[14] M. Yoon, S. Berber, and D. Tománek, Phys. Rev. B. 71, 155406 (2005).

[15] M. Hodak and L. A. Girifalco, Chem. Phys. Lett. 350, 405 (2001).

[16] M. Ashino, D. Obergfell, M. Haluska, S. Yang, A. N. Khlobystov, S. Roth, and R. Wiesendanger, Nature Nanotech. 3, 337 (2008).

[17] S. Berber, Y.-K. Kwon, and D. Tománek, Phys. Rev. Lett. 84, 4613 (2000). 